\title{
IDENTICAL TRIPLETS
}

*Mrs. S. Lavanya \& **Ms. J. Jayasakthi Prabhavathi

\section{INTRODUCTION}

\section{Multiple pregnancies have}

substantially higher risks of fetal mortality and morbidity than singleton pregnancies. The risks are more as the number of fetuses increase. With increase in number of assisted reproductive technology there have been raise in multiple pregnancy especially Higher Order Births. Multiples contribute to a large chunk of prematurity, low birth weight and NICU admissions. $1 / 5^{\text {th }}$ of all triplet pregnancies and one half of all quadruplets pregnancies results in at least one child with a major disability. Maternal problems are also more in multiple pregnancies.

\section{CASE REPORT}

A 26 year old primi gravida at 28 weeks with multiple gestations was admitted with the complaints of burning micturation, difficulty in breathing and discomfort. On admission the patient's BP was $140 / 90 \mathrm{~mm}$ of $\mathrm{Hg}$. Administered T. Depin 5mg. She was receiving regular antenatal care. Day before admission to the hospital she had complaints of cold, cough and itching over the abdomen. She was advised to take saline nebulisation and benzoyl proxide $2.5 \%$ for 3 weeks.

\section{On examination,}

- Physical examination findings revealed pale conjunctiva and ankle edema.

- The abdominal girth was increased to $106 \mathrm{cms}$, SFH increased and multiple fetal parts felt.

- Heart sound heard at two separate spots.

- USG examination revealed identical triplets (monochorionic diamnoitic and monochorionic monoamniotic fetus) with 30 weeks of gestation, anterior placenta and AFI $16 \mathrm{~cm}$.
- All blood (CBC, LFT, RFT and electrolytes) investigations were within normal limits.

- The urine culture reported presence of micro organisms. She developed UTI, Inj. Taxim $1 \mathrm{~g}$ bd and syp. Citralka was administered for 7 days.

The physician planned to wait for expectant management and intensive antepartum fetal surveillance. Two doses of Inj. Betamethasone $12 \mathrm{mgs}$ was administered Intra Muscularly at $24 \mathrm{hrs}$ apart. Mother crossed 34 weeks of gestation with great difficulty. The obstetrician planned for elective caesarean section at 34 completed weeks of gestation. The mother delivered three alive babies by LSCS under spinal anesthesia. Sex of all babies were male with the birth weight of $2.71 \mathrm{kgs}, 2.6 \mathrm{kgs}$ and $2.1 \mathrm{kgs}$ and APGAR score was 7/10, 9/10 for $1^{\text {st }}$ and $5^{\text {th }}$ min for all babies. The babies were shifted to NICU for observation and continuous monitoring.

\section{MEANING}

Triplets are known as Higher Order Multiples (HOM), which signified more than two babies are being carried out one time, although rare, it is entirely possible for women to conceive naturally with three babies; however fertility assistance is the strongest influential factor when it comes to have triplets. The current estimate is that around 80-90 sets of HOM are born in Australia each year.

Identical triplets are created when one egg is released and fertilized, but splits and then one half splits again.

\section{VARIETY OF TRIPLETS}

Fraternal triplets: when three eggs are released and each is fertilized by a different 
sperm or this may be occur during IVF fertility treatment.

Identical triplets: these are created when only one egg is released and fertilized, but splits and then one half splits again.

Mixed triplets: two identical and one fraternal occur when two eggs are released and fertilized separately, and one of these fertilized eggs splits again.

\section{FORMATION OF IDENTICAL TRIPLETS}

When one single egg is fertilized by one single sperm and this fertilized zygote quickly and evenly divides into three separate zygotes, identical triplets are formed. Another possibility is that the fertilized egg splits into 2 and then one of these eggs divides again. These are formally known as monozygotic triplets. This splitting occurs shortly after fertilization and before a woman even suspects she is pregnant.

\section{INCIDENCE}

The incidence of triplets and higher order multi fetal gestation in the US has risen an several $100 \%$ since 1980 , due to increasingly wide spread availability of fertility therapies. The natural incidence of spontaneous triplets pregnancy is approximately 1 in 6000 to 8000 births. By comparison, triplet and higher order birth accounted for 137 per 100,000 live births in 2011.

\section{CLINICAL FEATURES}

- Nausea and vomiting - extra babies can intensify the symptoms of morning sickness.

- Fatigue - excessive fatigue is one of the most commonly repeated complaints of mothers of triplets in the early pregnancy.

- Weight gain - On average, pregnant women with triplets gain 30 to 55 pounds.

- Abdominal size- Unusual rate of abdominal enlargement.
- Fetal movement-Excessive fetal movements may be notified by an experienced parous mother.

- Cardio respiratory embarrassment which is evident in later months - such as palpitation (or) shortness of breaths.

- Difficulty in walking.

\section{INVESTIGATION}

Women should be evaluated for ovulation induction drugs (or) use of ART and for family history. Triplet pregnancies are exclusively and accurately diagnosed by ultrasound examination. The chorionicity of the multiple gestation should be determined by USG. Monochorionic dichorionic placentation confers a higher risk of adverse outcome in both spontaneous conceptions and those conceived with ART. If they share a placenta, this is a strong and definitive sign of identical triplets. On examination, the abdomen is unduly enlarged, uterus is more than the period of amenorrhea, abdominal girth is increased and too many fetal parts is found. There are three distinct fetal heart sound located at separate spots.

Triples screening test does not work with triplets. USG helps to reveal some birth defects. The physician may also recommend amniocentesis or chorionic villi sampling in which tiny amount of tissues from placenta is removed and examined.

\section{COMPLICATIONS}

\section{Maternal complications}

- Anemia

- Hypertension And Pre Eclampsia

- Placenta Previa

- Hydrominos

- Antepartum Hemorrhage

- Gestational Diabetes

- Preterm Labour And Delivery

- Post Partum Hemorrhage

- Post Op Complications 


\section{PROGNOSIS}

Maternal mortality is increased in multiple pregnancy due to hemorrhage, Pre eclampsia and anemia. Maternal morbidity is due to prevalence of complications and increased operative interference. Perinatal mortality is high due to pre maturity, is extremely high in monozygotic.

\section{MANAGEMENT:}

ANTENATAL MANAGEMENT;

- Prompt treatment for morning sickness

- More frequent antenatal visits

- Complete bed rest

- Diet - triplet women need to consume 200 gms of protein daily, enough iron, calcium, folic acid and other nutrients.

- Regular USG examination.

- Assessment of fetal well being.

MULTI FETAL AND SELECTIVE PREGNANCY FETAL REDUCTION

Selective fetal reduction is needed when fetus in a multiple gestation is abnormal. Multiple fetal reductions is done in higher order pregnancies to minimize the inherent complication like pre term labour and to improve the outcome of pregnancies.

INTRANATAL MANAGEMENT Best way to deliver triplets is caesarean section because the babies are - Miscarriage Rate Is Increased

- Higher Incidence Of Congenital Anomalies - Pre Maturity- Majority Of Triplets Born Before 34 Weeks Of Gestation

- Low Birth Weight - Average Birth Weight For Individual Triplet Around 1.8kgs

- Respiratory Distress Syndrome due to Under Developed Lungs

- Higher Risk Of Infection

- Transfusion Syndrome between Triplets

- Cerebral Palsy

- Delay in Development. - IUGR
The risk of PPH can be minimized by routine administration of Inj. Methergin $0.2 \mathrm{mg}$ and Inj.Oxytocin infusion. If blood loss is more replace by blood transfusion. The patient is carefully watched for 2 hours after delivery. After the birth, the babies need to shift directly to NICU. Premature triplets have similar risk and problems as single or twin babies who are premature. Postnataly observe the mother's vital parameters, hemorrhage and post op complications.

NURSES RESPONSIBILITY:

- Provide comfort and complete bed rest.

- Assessment of well being of the fetus

- Advise the mother for regular antenatal check

- Plan for antenatal diet to meet the requirement of the triplets.

- Regularly reassure the mother to reduce fear anxiety. PIH, GDM.

- Provide Pre and post operative care.

\footnotetext{
not positioned properly for vaginal delivery.
} 
INTERESTING

MULTIPLES:

- Worldwide there are at least 125 million living multiples.

- Did you know that up to $22 \%$ of twins are left handed.

- Identical twins exhibit almost identical brain wave patterns.

- The average time between the delivery of the $1^{\text {st }}$ and second twin is 17 minutes

- Nigeria has the highest rates of multiples with more identical twins than fraternal.

- William shakesphear was the father of boy/girl twin.

- In 2004 about 1 in 34 babies was born a twin or triplet in UK (1980 1 in 52).

- Incidence of quadruplets is 1 in 142 births. CONCLUSION

The essence of successful outcome of a multiple pregnancy is to make an early diagnosis. Information and support from well informed health care professional is required for a woman who has been diagnosed as multiple pregnancy which will help to prepare the parents and to prevent potential complications. Careful monitoring and observation will improve the Prognosis.

\section{REFERENCE:}

Helen Varney,(1987) Nurse Midwifery edition 2, Pg. no. $180-181$.

Linda V. Walsh,(2001) Midwifery Community Based Care during Child Bearing Year, pg. no. $240-257$

Krishna, Jank, Daftary(2001) Pregnancy at risk current Concepts, Edition 4, Pg. No. 143

Sheila Balakrishnan,(2012) Text book of Obstetrics, edition 2 Pg No. $176-178$

Adele Pillitteri,(2007) Maternal and Child Health Nursing Pg. No. $434-435$.

Susan Mattson and Judy E. Smith, (2004) Maternal - Newborn Nursing, $3^{\text {rd }}$ edition, pg. No. $831-835$ 